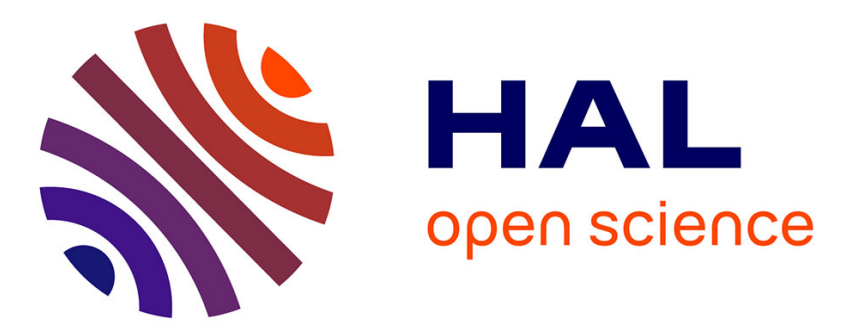

\title{
The influence of ion implantation and high energy proton irradiation of semiconductors on a photothermal signal
}

\author{
A. Glazov, K. Muratikov
}

\section{- To cite this version:}

A. Glazov, K. Muratikov. The influence of ion implantation and high energy proton irradiation of semiconductors on a photothermal signal. Journal de Physique IV Proceedings, 1994, 04 (C7), pp.C7163-C7-166. 10.1051/jp4:1994739 . jpa-00253268

\section{HAL Id: jpa-00253268 https://hal.science/jpa-00253268}

Submitted on 1 Jan 1994

HAL is a multi-disciplinary open access archive for the deposit and dissemination of scientific research documents, whether they are published or not. The documents may come from teaching and research institutions in France or abroad, or from public or private research centers.
L'archive ouverte pluridisciplinaire HAL, est destinée au dépôt et à la diffusion de documents scientifiques de niveau recherche, publiés ou non, émanant des établissements d'enseignement et de recherche français ou étrangers, des laboratoires publics ou privés. 


\title{
The influence of ion implantation and high energy proton irradiation of semiconductors on a photothermal signal
}

\author{
A. Glazov(1) and K. Muratikov(1) \\ Friedrich-Schiller-University Jena, Institute for Optics and Quantum Electronics, Max-Wien Platz I, \\ 07743 Jena, Germany
}

\begin{abstract}
A photothermal technique with interferometrical detection was applied to obtain amplitude and phase images of ion-implanted and proton-irradiated semiconductors. It was experimentally shown that ion implantation and radiation defects differently affect on the photothermal images of semiconductors by using weakly absorped laser radiation for heating. Oscillations observed by photothermal imaging was explained by interference of the heating light due to internal reflection and should be taken into account for right interpretation of the images.
\end{abstract}

\section{INTRODUCTION}

Photothermal methods are very promising for local nondestructive evaluation in semiconductor technologies at various stages, a measurement of optical, thermal and electronic properties of semiconductors and determination of both surface and bulk structures. Over the past few years, a number of these techniques have been shown to be effective for ion implantation investigation [1,2]. In this case, photothermal methods provide higher sensitivity as compared to conventional techniques (such as Rutherford backscattering or Raman spectroscopy). On the other hand, irradiation of semiconductor materials, structures, and devices is of great importance in modern technology and physics, and it is very attractive to apply the photothermal technique to its measurement.

Here we discuss results of some measurements of various semiconductor wafers with defects produced by both ion implantation and proton-irradiation and their influence on a photothermal signal.

\section{EXPERIMENTAL RESULTS}

To examine both ion-implanted and proton-irradiated semiconductors a recently developed photothermal technique with mirage effect arrangement and interferometrical detection [3] was applied.

Generation of thermal waves in samples was provided by a cw-modulated laser with a wavelength of $1.06 \mu \mathrm{m}$. The maximal power of the light flux at the sample surface was about $100 \mathrm{~mW}$. A modulation frequency of the pump radiation during the experiments ranged from 125 to $10^{5} \mathrm{~Hz}$. The variation of a refraction index of adjacent air over the surface due to heating a sample was detected by the He-Ne laser with an output power of $8 \mathrm{~mW}$. A probe beam waist at the center of an induced thermal lens was about $30 \mu \mathrm{m}$, whereas the distance between the surface and a beam axis was approximately $50 \mu \mathrm{m}$. The interferometrical detection was used to decrease the influence of acoustic noises at low modulation

(1) Permanent address: A.F. Ioffe, Physicotechnical Institute of the Academy of Sciences of Russia,

Politehnicheskaya 26, 194021 St. Petersburg, Russia 
frequencies and, therefore, to decrease measurement time. The experimental setup provides $2 \mathrm{D}$ amplitude and phase images with a scanning rate about $0.03 \div 0.1 \mathrm{f}$ points/s, where $\mathrm{f}$ is the modulation frequency in $\mathrm{Hz}$.

Figure 1 presents typical ID amplitude image of an InP wafer partly implanted with $90 \mathrm{keV} \mathrm{Mg}$ ions to the dose of $10^{13} \mathrm{~cm}^{-2}$. One can see a sharp increase of the photothermal amplitude in scanning the implanted region, accounted for by stronger absorption of the pump radiation in the damaged subsurface layer. It should be noted that the measurements were made directly after implantation with no subsequent anneal. The photothermal amplitude image in figure 1a shows also considerable oscillations. An oscillation period varies for different samples and scan directions. However, their amplitude was usually considerable and about $20-40 \%$ of the signal amplitude at all modulation frequencies.

These oscillations result from the optical interference of the heating light reflected from a back surface, because the sample had a polished one. The interference taking place in highly absorbing region of implanted side, accounts for changing an effective power of the heating light and, therefore, for changing a corresponding contribution to the photothermal signal. In this case, a standing light wave appears in the sample. The oscillations in images are due to a nonparallelism of the surfaces. When scanning along the wafer, a thin subsurface layer contributing to the thermal wave signal locates alternately at either the loop or the node of the standing light wave because of a wafer thickness nonuniformity. This leads to a periodical change of absorbed light energy in the sample scan, while the average energy absorbed in the bulk is constant and does not depend on the sample thickness. So, the oscillating component associated with the surface absorption appears against the background of a uniform contribution due to bulk absorption. The analysis of the signal phase behavior shows that the photothermal signal from implanted regions of InP wafers mainly results from the surface absorption at $1.06 \mu \mathrm{m}$ wavelength and the bulk absorption contribution is negligible. It should be noted that a degree
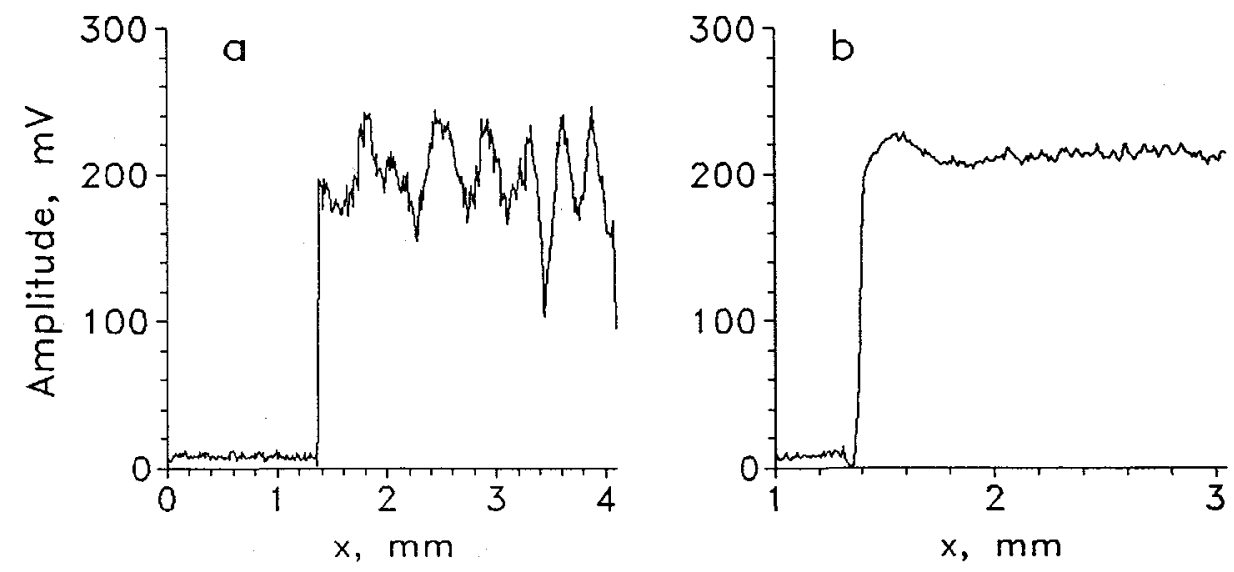

Fig.1. 1D amplitude image of an InP wafer. Implanted region $x>1.4 \mathrm{~mm}$. a) for a sample with both polished surfaces. b) for the same sample with a rough back surface.

of the wafer surface nonparallelism may be determined from the measurement of the oscillation period. The images of the same wafers with roughed back surface have no oscillation (figure 1b).

The image without oscillation allows to observe special features of a photothermal signal behavior near 
the border between implanted and nonimplanted regions of the wafer. Namely, there are a maximum at the side of implanted region and a minimum at the opposite side. Similar minimum and maximum were obtained by simulation for a sample with a semi-infinite subsurface layer with higher thermal diffusivity and result from thermal wave interference near the border. These features point out that inhomogeneity takes place not only for optical absorption but for thermal properties of the sample also.

Similar photothermal images were obtained in scanning higher absorbing GaAs and Si wafers [4]. However, in the case of GaAs samples with polished surfaces, the oscillations take place also in phase images owing to simultaneous contributions of surface and bulk absorption.The oscillations in images of Si wafers were much smaller due to high bulk absorption of the pump radiation.

We have measured also high-energy-proton irradiation of semiconductors. Figure 2 presents 2D photothermal images of GaAs wafers irradiated with fast protons with the energy of $6.7 \mathrm{MeV}$ to the dose of $3 \cdot 10^{12} \mathrm{~cm}^{-2}$ [5]. Unlikely the case of ion implantation, the oscillations take place only for unexposed region $(x<3 \mathrm{~mm})$. A phase image has the same features. Figure 2a shows that the irradiation leads to a destruction of interference pattern. The reason is likely a scattering of the reflected light from the back surface by produced radiation defects in the bulk. The images of the wafers with a rough rear surface demonstrate no oscillation, and one can see small changes of the amplitude of the photothermal signal when crossing a border of the irradiated region. These changes do not exceed $10 \%$ of the signal magnitude and several degrees for the signal phase and were caused by an increase of heating light absorption in the bulk, because the surface of the sample was optically homogeneous. A fine structure of the images is reproducible and takes place not owing to a time noise, rather to a weak spatial inhomogeneities of the wafer.

The amplitude of the oscillations allows to estimate relative contributions of surface and bulk absorption to the photothermal signal. Assuming that the part of pump light reflected from the back surface and absorbed at the front one consists about $10 \%$ of total light absorbed at the surface, as the analysis of the image of ion-implanted GaAs wafer shows, one can say that the surface absorption contribution to the photothermal signal at the modulation frequency of $1 \mathrm{kHz}$ consists $3 \%$ of the bulk absorption contribution. That is why the oscillations take place also in the phase photothermal images.

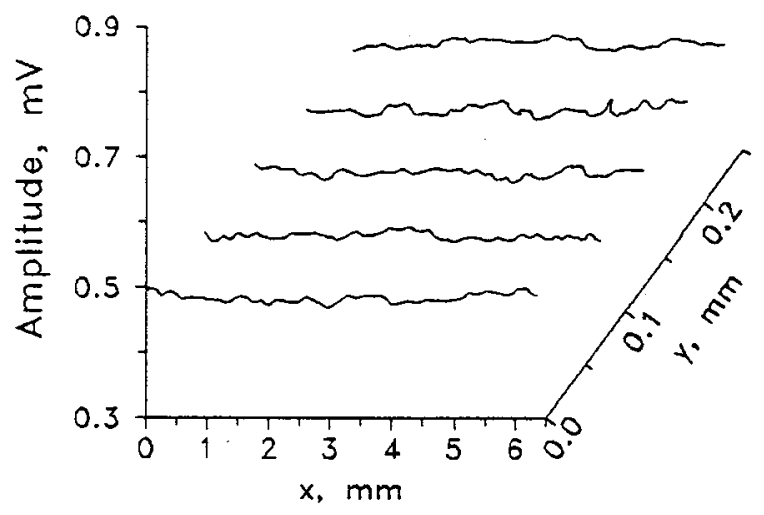

Fig.2. 2D amplitude image of an GaAs wafer. Irradiated with $\mathrm{p}^{+}$region is $\mathrm{x}>3 \mathrm{~mm}$. a) for a sample with both polished surfaces. b) for the same sample with a rough back surface.

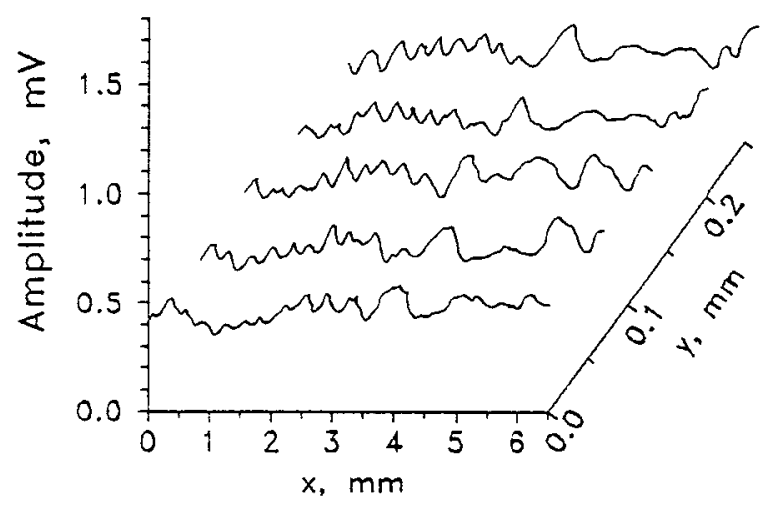




\section{CONCLUSION}

To conclude, the photothermal interferometrical technique in the mirage effect geometry was shown to be useful to locally test radiation defects and ion implantation into various semiconductors. It was found that the interference of the pump radiation due to the reflection from a rear surface influences the photothermal images of weakly absorbing semiconductor wafers. For instance, this results in spatial oscillations of the photothermal signal in the case of a nonuniform wafer thickness. A neglect of the effect can leads to incorrect interpretation of the images and to errors of a measurement of object parameters, for example, an implantation dose.

The investigations show also that there are significant differences between the mechanisms of interaction of intraband radiation with ion-implanted semiconductors and semiconductors irradiated with high-energy protons. In the former case the absorption in the surface layers increases significantly, while in the latter case strongly-scattering defects appear. In order to determine the effect of proton irradiation on the thermal wave signal with only one polished face, similar experimwents should be performed with longerwavelength exitation laser radiation.

\section{ACKNOWLEDGMENTS}

The authors would like to thank Dr. H.-G. Walther for the fruitful discussion.

\section{References}

[1] Mandelis A.(ed.), Photoacoustic and Thermal Wave Phenomena in Semiconductors (New York, Amsterdam, London: North-Holland, 1987).

[2] Smith W.L., Rosencwaig A., and Willenborg D.L., Appl. Phys. Lett. 47 (1985) 584-586.

[3] Glazov A.L. and Muratikov K.L., Int. J. of Optoelectronics 4 (1989) 589-597.

[4] Glazov A.L., Muratikov K.L., and Suvorov A.V., Appl. Phys. Lett. 61 (1992) 569-571.

[5] Glazov A.L., Kalinovskii V.S., and Muratikov K.L., Sov. Tech. Phys. Lett. 1 b (1992) 132-134. 